\section{OFF-PUMP CORONARY ARTERY BYPASS GRAFT (OPCABG) SURGERY IN PATIENTS WITH LEFT MAIN CORONARY ARTERY STENOSIS: ANALYSIS OF EXPERIENCE IN 145 PATIENTS}

\author{
Mohammad Fazle Maruf ${ }^{1}$, Suman Nazmul Hosain², Md \\ Kamrul Hassan ${ }^{1}$, Zahangir Haider Khan ${ }^{3}$, Asif Ahsan \\ Chowdhury $^{4}$, Tahmina Akter ${ }^{4}$, Rezaul Karim ${ }^{4}$, NA Kamrul \\ Ahsan $^{5}$
}

\begin{abstract}
:
The presence of significant left main arterial stenosis has been considered a relative contraindication to OPCAB surgery. The development of newer techniques and an increasing understanding of the hemodynamic changes make the surgeons confident to perform OPCAB in patients with significant left main artery stenosis. Between January 2004 to March 2008, 145 patients with left main artery stenosis underwent OPCAB surgery at NICVD. Gradual adoption and integration of maturing heart surgery in fast years led to a trend towards overall improvement in CABG outcomes. Offpump coronary artery surgery appears to be a safe and effective technique in patients with significant Left Main coronary artery disease. The postoperative morbidity and length of ICU and hospital stay are shorter. OPCAB could be an effective alternative to the conventional method CABG with same or better early results and is cheap and cost effective. The longterm results are to be evaluated
\end{abstract}

\section{Introduction:}

Since the first clinical description of left main coronary artery disease by Herrick in 1912, studies showed that stenosis of the left main coronary artery is of critical prognostic importance. CABG surgery lessens symptoms and significantly prolongs survival.

CABG is indicated in patients with significant left main coronary artery stenosis ( $>50 \%)^{1}$ and surgery prolongs life among patient with left main coronary artery disease in comparison to medical therapy. ${ }^{2}$ Because of increased focus of on less invasive surgery and development of enabling technology and technique Off-pump Coronary Surgery (OPCAB) is reintroduced in the past few years and now account for nearly 20\% CABG operations in US and western Europe ${ }^{3}$. Cardiac Surgeons accept less favorable operative exposure to operate on beating heart with the idea that avoiding CPB improves end-organ function eliminating the problem of activation of inflammatory cascades and trauma.

1. Assistant Professor, Cardiac surgery, NICVD.

2. Associate Professor, Cardiac Surgery, NICVD.

3. Resident Surgeon, NICVD

4. Assistant Registrar, Cardiac Surgery, NICVD.

5. Professor \& Head, Dept. of Cardiac Surgery, NICVD
Minimally invasive direct coronary bypass (MIDCABG) and off-pump coronary bypass (OPCABG) have eliminated one major component of aberrant physiology intrinsic to on-pump coronary bypass procedures. Coronary bypass, without the use of cardiopulmonary bypass (CPB) has been shown to reduce the overall systemic inflammatory response, including cytokinemediated responses ${ }^{4}$. Furthermore, cardioplegic arrest adds an additional level of physiologic derangement directly upon the heart and troponin I release and myocardial injury are reduced in off-pump procedures ${ }^{4,5}$. Elimination of CPB would lead to more physiologic milieu that would encourage optimal organ function especially heart during and immediately following coronary revascularization and this might reduce complications especially in left main arterial stenosis, a high risk subgroup.

The presence of significant left main arterial stenosis has been considered a relative contraindication to OPCAB surgery. The development in exposure and stabilization techniques, the introduction of intra-coronary shunts, and an increasing understanding of the hemodynamic changes which occur during off-pump surgery make the surgeons confident to perform OPCAB in patients with significant left main artery stenosis ${ }^{6}$.Although a few small-randomized studies have demonstrated a benefit from Off-pump, a preponderance of retrospective analysis has mortality and morbidity benefit in specific Subgroup ${ }^{2}$. Our aim in this study was to analyze retrospectively the outcomes in patients who underwent offpump CABG for left main disease.

\section{Materials \& Method:}

\subsection{Patients selection}

National Institute of Cardiovascular diseases and Hospital is tertiary referral centre and accepts patients referred with coronary artery diseases. In this study Retrospective review of non-randomized prospectively collected data showed that 145 patients with left main artery stenosis underwent OPCAB surgery among the 243 patients of LMS receiving coronary artery bypass surgery in NICVD between January 2004 to March 2008 , all those who met the following criteria were selected in a nonrandomized manner and included in the study:

- The presence of LMCA stenosis, as defined by a reduction of the arterial lumen by more than $50 \%$, isolated or accompanied by stenosis of other coronary arteries.

- Patients with suitable anatomy of epicardial non-calcified vessel $>1 \mathrm{~mm}$.

- Patients without evidence of moderate to severe cardiomegaly.

- Patients with no intramyocardial target vessel.

- Patient with no cardiac arrhythmia.

- No myocardial revascularization done with other associated procedures (such as valve replacement, aneurysm resection and so on. 


\subsection{Surgical procedures:}

CABG in left main coronary artery disease were performed through either median sternotomy or left lateral thoracotomy under general anesthesia \& Left Internal Thoracic Artery (LITA) harvested. Deep pericardial sling and mechanical device archin, starfish were used to position the heart for optimal visualization of the lateral, posterior, inferior and the distal right coronary arteries. To maintain hemodynamic stability, at times patients were placed in trendelenburg position for grafting PDA, and OM. Stabilization of the target vessels were done with Octopus 3 stabilizer. We used tape proximally to control flow at the anastomotic site and also used mist air blower to clear the surgical field. LITA was almost always anastomosed to the LAD as a pedicle graft. The sequence of grafting was individualized for each patient, depending on the severity of the lesions in different coronary arteries and the patient's hemodynamics. Intracoronary shunts were used. Proximal anastomoses were made to ascending aorta with side clamp.

\section{Results:}

Table-I shows the preoperative data of the population. The patient population consisted of 14 women and 135 men (total 145 ), mean age $55 \pm 11$ years(range25-68years). Angina pectoris was the presenting symptom in 142 patients and only 30 patients presented with exercise induced dyspnea.36 patients (24.8\%) had history of preoperative myocardial infarction.

Table-I: Patients characteristics of Leftmain coronary artery stenosis

\begin{tabular}{lc}
\hline Characteristics & Number of patients n=145 \\
\hline Age in years (mean \pm SD & $55 \pm 11$ \\
Female & $14(9.65 \%)$ \\
Pre op MI & $36(24.8 \%$ \\
Smokers & $71(48.96 \%)$ \\
Diabetes & $53(36.55 \%)$ \\
Hypertension & $42(28.96 \%)$ \\
Dyslipidemia & $64(44.14 \%)$ \\
Renal dysfunction & $5(3.49 \%)$ \\
H/o CVA & $6(4.14 \%)$ \\
PVD & $13(8.96 \%)$ \\
Chronic Lung D. & $18(12.41 \%)$ \\
CCS(I-II) & $54(37.24 \%)$ \\
CCS(III-IV) & $88(60.69 \%)$ \\
Dyspnea & $30(20.7 \%)$ \\
Lt. Main only & $31(21.38 \%)$ \\
1-2 VD+ Lt. Main & $84(57.93 \%)$ \\
3 VD+ Lt. Main & $30(20.69 \%)$ \\
LVEF & $49.2 \pm 10.6$ \\
\hline
\end{tabular}

MI: Myocardial infarction; CVA: cerebrovascular accident; PVD: peripheral vascular disease; CCS: Canadian Cardiovascular Score; LVEF: left ventricular ejection fraction; Preop: preoperative.
The average number of distal anastomoses per patient were 2.4 \pm 0.7(Table-2).Coronary artries grafted were LAD 145 (100\%), RCA/PDA 69 (47.59\%), Diagonal 20(13.79\%),

Marginal branches 103 (71.03\%), LCX main 3 (2.07\%). Regarding the type of grafts, the majority of patients received a combination of left internal mammary artery and saphenous veins (Table-II). Median sternotomy was employed 129(90.35\%) and the so-called MIDCAB through a small left anterior thoracotomy was used in 14 (9.65\%) patients..

There were 4 incidences of intraoperative conversion from off-pump to on-pump surgery One patient with history of failure and with enlarged heart were converted to on pump, because of electrical instability\& other three were for hemodynamic instability. The mean intubation period, intensive care unit stay and postoperative hospital stay were $5.4 \pm 2.2$ hours, $48 \pm 6$ hours and $6 \pm 4$ days, respectively. There were 4 hospital deaths ((2.76\%). Perioperative myocardial infarction was observed in $4(2.76 \%)$ patients, postoperative stroke in $1(0.69 \%)$, prolonged ventilator support in 6 (4.14\%) and mediastinitis in 3 (2.07\%).

Table-II: Intraoperativevariables of patients with Left main coronary artery stenosis

\begin{tabular}{|c|c|}
\hline Variables & Number of patients $n=145$ \\
\hline Conversion to ECC & $4 \quad(2.76 \%)$ \\
\hline Distal anastomosis/patient & $2.4 \pm 0.7$ \\
\hline \multicolumn{2}{|l|}{ Types of conduits } \\
\hline LITA & 145 (100\%) \\
\hline Long saphenous vein & 203 \\
\hline \multicolumn{2}{|l|}{ Bypassed arteries } \\
\hline LAD & $145(100 \%)$ \\
\hline Diagonal & $20(13.79 \%)$ \\
\hline Ramus Intermedius & $8 \quad(5.51 \%)$ \\
\hline RCA/PDA & $69 \quad(47.59 \%)$ \\
\hline $\mathrm{OM}$ & $103(71.03 \%)$ \\
\hline LCX main & $3(2.07 \%)$ \\
\hline ECG change & $2(1.38 \%)$ \\
\hline Hemodynamic change & $4(2.76 \%)$ \\
\hline Inotropic support & $25 \quad(17.24 \%)$ \\
\hline Blood transfusion & $1.85 \pm 1.42$ unit \\
\hline Intraoperative IABP & 0 \\
\hline Total operating time (min) & $182 \pm 47$ \\
\hline MIDCAB & 14 (9.65\%) \\
\hline
\end{tabular}

LIMA: Left internal mammary artery; RIMA: right internal mammary artery; CPB:cardiopulmonary bypass; AoXL: aortic cross clamp; RA: radial artery; IABP: intra-aortic balloon pump; NA: not applicable 
Table-III : Postoperative outcome of patients with Left main stenosis

\begin{tabular}{lc}
\hline Variables & Number of patients \\
\hline Perioperative MI (\%) & $4(2.76 \%)$ \\
Reoperation for bleeding (\%) & $2(1.38 \%)$ \\
Postoperative inotropes (\%) & $24(16.55 \%)$ \\
Blood loss (ml/24h )mean \pm SD & $378 \pm 20$ \\
Blood transfusion (unit) & $1.72 \pm 1.42$ \\
Atrial fibrillation (\%) & $128.27 \%$ \\
Intubation time in hours & $5.4 \pm 2.2$ \\
Prolonged ventilation (\%) & $6(4.14 \%)$ \\
Postop IABP (\% & 0 \\
Stroke (\%) & $1 \quad(0.69 \%)$ \\
Pulmonary complications (\% & $19(13.1 \%)$ \\
Mediastinitis (\%) & $3(2.07 \%)$ \\
Acute renal failure (\%) & $1(0.69 \%)$ \\
Hospital mortality (\% & $4(2.76 \%)$ \\
ICU stay in hours (mean $\pm S D)$ & $48 \pm 6$ \\
\hline
\end{tabular}

MI: myocardial infarction; IABP: intra-aortic balloon pump; ICU: intensive care unit;

Postop: postoperative

\section{Discussion:}

Significant left main coronary artery stenosis is a standard indication for coronary artery bypass grafting regardless of the patient's presenting symptoms. Numerous studies ${ }^{2,3}$ have showed that Surgical revascularization prolongs life in patients with significant LMCA stenosis compared to medical therapy alone. Left main coronary artery disease is an independent predictor of postoperative morbidity and mortality in patients undergoing coronary revascularization. . The presence of critical LMCS has been considered a relative contraindication for the off-pump coronary artery bypass (OPCAB) technique due to concerns over the well demonstrated hemodynamic changes during displacement of the heart.

With the perfection of techniques and equipments for Stabilization the clinical benefit of' off-pump CABG (OPCAB) in left main coronary artery stenosis became more apparent.

Revascularization of the circumflex territory is in most cases difficult because of hemodynamic impairment associated with exposing the vessel. With availability to apply stabilization techniques the adoption rate of our multi-vessel beating heart surgery of left main coronary artery stenosis has increased. We found use of Starfish/Urchin stabilization along with verticalization of the apex provides an excellent haemodynamic toleranance.

This retrospective review of prospectively collected data demonstrates that patients with significant left main coronary artery stenosis can be revascularized safely using off-pump techniques. The degree of stenosis was not stratified, and patients were not selected for revascularization either on-pump or off-pump based on the degree of left main coronary artery occlusion.

In our series the mean number of grafts was $2.4 \pm 0.7$ (Table-II) which is similar to other studies ${ }^{11,12}$. And in large reported series analysis number of graft shows no significant difference from Conventional groups with CPB.

The result of this study suggest that myocardial revascularization in the presence of left main coronary artery stenosis can be done safely with low early mortality and morbidity.

Although this is a Small series and early experience, this observation is similar with other worker ${ }^{2}$. The overall lower incidence of complication in the reports particularly neurological (0.69\%), Peri-MI (2.76\%) of OPCAB .

Patients with significant LMCS present a special situation because the hemodynamic disturbances which develop during manipulation of the heart in OPCAB surgery may not be tolerated by the patient. In our experience, LIMA-LAD anastomosis can be performed without much displacement of the heart, and the use of an intracoronary shunt to permit continued myocardial perfusion during anastomosis reduces hemodynamic disturbances to a minimum. Left anterior descending artery grafting using LIMA via left anterior small thoracotomy without CPB has been shown to offer good early and mid-term results. ${ }^{14}$

Access to the obtuse marginal and posterior descending artery needs displacement and manipulation of the heart. Various techniques have been described to access and revascularize the vessels on the lateral and inferior walls of the ventricle without compromising hemodynamics significantly ${ }^{18}$.Cartier et al. ${ }^{19}$ described a technique of exposing the circumflex vessels. They used four pericardial sutures around the base of the heart and then used a pull-type mechanical stabilizer. Using this technique, they could easily access the obtuse marginal as well as the posterolateral branches while maintaining hemodynamic stability. They achieved complete revascularization in 95\%of patients with one patient needing conversion to cardiopulmonary bypass. We used a combination of Octopus, Starfish/Urchin stabilization along with verticalization of the apex provides an excellent haemodynamic toleranance ,deep pericardial sutures, Trendelenburg position, right pleurotomy and vertical pericardiotomy near the diaphragm to expose the circumflex vessels. The majority of patients were moved out of the ICU within 24 hours.

In our study we observed lower postoperative morbidity, particularly perioperative MI, blood loss, requirement of blood 
or blood products and atrial fibrillation. The ICU and hospital stay were shorter in this group. There was a definite trend toward improved in -hospital survival of OPCAB patients with LMCAS. Eliminating CPB in coronary revascularization reduces the incidence of intraoperative blood transfusion requirements. Our study supports obviating the need for CPB and cardiologic arrest may improve clinical outcomes particularly in left main artery disease patient.

In conclusion, although the fear of CPB hazard that led to beating procedure is still debated ${ }^{5}, 3$, gradual adoption and integration of maturing heart surgery in fast years led to a trend towards overall improvement in CABG outcomes.

Off-pump coronary artery surgery is a safe and effective technique in patients with significant Left Main coronary artery disease. The postoperative morbidity and

length of ICU and hospital stay are shorter. OPCAB Can be an effective alternative to the conventional method CCABG with same or better early results and is cheap and cost effective. The long-term results are to be evaluated.

\section{References:}

1. Herrick J.Clinical features of sudden obstruction of the coronary arteries.JAMA 1912;59:2015-20.

2. Frank W. Sellke, Pedro J .del Nido, Scott J. Swanson. Sabisston \& Spencer Surgery of the Chest. $7^{\text {th }}$ ed.Elsvier Saunders,Philadelphia 2005;vol 2:P-1461.

3. Chaitman BR, Fisher LD,Bourassa MG,Davis K Rojers WJ,Tyras DH. Effect of Coronary bypass surgery on survival patterns in subsets of patients with left main coronary artery disease. Report of the collaborate study in coronary artery surgery(CASS) Am J Cardiol.1981;48:765-77.

4. Wan S,Izzat MB, Lee TW, Wan IYP, Tang NLS, Yim APC.Avoiding cardiopulmonary bypass in mulivessel CABG reduces cytokine response and myocardial injury. Ann Thorac Surg 1999;68:52-7.

5. Struber M, Cremer JT, Gohrabandt B, etal.Human cytokine responses to coronary artery bypass grafting with and without cardiopulmonary bypass. Ann Thorac Surg 1999;68:1330-5.

6. Yeatman M,Caputo M.Ascione R,Ciulli F,Angelini GD.Off-pump coronary artery bypass surgery for critical left main stem disease:safety,efficacy and outcome.Eur J Cardiothorac surg.2001;19:239-242.
7. Mack MJ. Pro: beating heart surgery for Coronary revascularisation: in the most important development since the introduction of the heart lung machine? Ann Thorac Surg. 2000; 70:1774.

8. Mack M, Bachand D,RN et al. Improved outcome in coronary artery bypass grafting with beating heart technique. J. Thorac Cardiovasc. Surg. 2002; 124: 598.

9. Chang $\mathrm{W}$ et al, Off-pump coronary surgery; effect on early mortality and stroke. J.Thorac Cardiovasc Surg. 2002; 124: 313.

10. Ricci $M$ et al. Stroke in Octogenarian undergoing CABG with or without CPB. Ann Thorac Surg. 2000; 69:1471.

11. Calafiore AM. ct al.Myocardial revascularization with or without Cardiopulmonary bypass in multi vessel disease: impact of the strategy on early outcome. Ann Thorac.Surg. 2001; 72:456-63.

12. Takaro T,Peduzzi p,Detre KM,et al. Survival in subgroups of patients with leftmain coronary artery disease.Circulation1982;66:14-22.

13. Dewey TM ,Magee MJ, Edgerton JR ,Mathison M, Tennison D, Mack MJ. Off-pump bypass grafting is safe in patients with left main coronary disease. Ann Thorac Surg. 2001; 72: 788-792.

14. A. Coulson,SA Bahshay,Tj Sloon,1998,'Minimally invasive reoperation through a lateral thoracotomy for circumflex coronary artery bypass. Tex heart inst $\mathrm{J} ; 25(3): 170-4$.

15. A.P. Andrew, V.A.hunaid,M.J. Mask,G.A.Jullien,2006’ Nonanastomotic avulsion of a left internal mammary artery graft- a rare complication of minimally invasive direct coronary artery -bypass surgery.Intact Cardiovasc thorac Surg;5:454-455.

16. Brian Glenville.1999,'minimally invasive CardiacSurgery. BMJ; vol-319:135-136.

17. Michael A .Wait, 2000.'Treatment of Coronary heart disease with minimal surgery.Proc(Bayl Univ Med Cent);V13(2):121-127.

18. A. Coulson,SA Bahshay,Tj Sloon,1998,'Minimally invasive reoperation through a lateral thoracotomy for circumflex coronary artery bypass. Tex heart inst J;25(3):170-4.

19. Cartier R, Blain R. Off-pump revascularization of the circumflex artery: technical aspect and short-term results. Ann Thorac Surg 1999; 68: 94-98 